\title{
Relación entre el uso de dispositivos tecnológicos y la somnolencia diurna. Un estudio asociado al rendimiento académico en adolescentes
}

\section{Relationship between the use of technological devices and daytime sleepiness. A study associated with academic performance in adolescents}

http://dx.doi.org/10.17981/cultedusoc.12.2.2021.13

Recibido: 24 de noviembre de 2020 aceptado:16 de febrero de 2021 Publicado: 1 de julio de 2021.

\author{
Francis Araque-Barboza (1) \\ Universidad Metropolitana. Barranquilla (Colombia) \\ tita42_fa@yahoo.es \\ Elisama Beltrán-De-La-Rosa \\ Universidad Metropolitana. Barranquilla (Colombia) \\ elisamabeltran@gmil.com \\ Olaiza Lobato-Pérez (D) \\ Universidad Metropolitana. Barranquilla (Colombia) \\ olaiza.lobato@gmail.com
}

Para citar este artículo:

Araque-Barboza, F., Beltrán-De-La-Rosa, E. y Lobato-Pérez, O. (2021). Relación entre el uso de dispositivos tecnológicos y la somnolencia diurna. Un estudio asociado al rendimiento académico en adolescentes. Cultura, Educación y Sociedad, 12(2), $223-240$. DOI: http://dx.doi.org/10.17981/cultedusoc.12.2.2021.13

\section{Resumen}

El uso de dispositivos tecnológicos en estudiantes en la franja nocturna se relaciona con el rendimiento académico, de manera que el objetivo de este artículo es analizar esta problemática que al, desplazar el sueño se evidencia somnolencia diurna, afectando el rendimiento académico en los adolescentes. El estudio se desarrolla con una muestra de 201 estudiantes de secundaria de una Institución Educativa en Valledupar, Departamento Cesar, Colombia. El enfoque teórico-conceptual usado es multidisciplinario. El paradigma es cuantitativo, con un diseño transeccional, no experimental de corte correlacional. Entre los hallazgos destacan que un $68,2 \%$ de la población presentó niveles de somnolencia al utilizar dispositivos tecnológicos entre 3 y 6 horas después de las 7.00 p.m. Los estudiantes que declararon, siempre presentar sueño en el aula, tienen un rendimiento académico por debajo de los que respondieron no me da sueño. Se concluye en la necesidad de implementar estrategias de monitoreo y modelamiento de las prácticas de uso adecuado de los dispositivos tecnológicos en adolescentes con la participación de sus padres, dado que se identificó, una tendencia al cambio del cronotipo matutino a vespertino, lo cual tiene efectos neuropsicológicos colaterales.

Palabras clave: Dispositivos; tecnología; somnolencia diurna; rendimiento académico; adolescencia

\section{Abstract}

The use of technological devices in students in the night time slot is related to academic performance, so the objective of this article is to analyze this problem that by displacing sleep, daytime sleepiness is evidenced, affecting academic performance in adolescents. The study is developed with a sample of 201 high school students from an Educational Institution in Valledupar, Cesar Department, Colombia. The theoretical-conceptual approach used is multidisciplinary. The paradigm is quantitative, with a transectional, non-experimental, correlational design. The findings include that $68.2 \%$ of the population presented levels of drowsiness when using technological devices between 3 and 6 hours after 7:00 p.m. The students who declared that they always present drowsiness in the classroom have an academic performance below those who responded, I don't feel sleepy. It is concluded on the need to implement strategies for monitoring and modeling the practices of adequate use of technological devices in adolescents with the participation of their parents, given that a tendency to change the chronotype from morning to evening was identified, which has effects collateral neuropsychological.

Keywords: Devices; technology; day time sleepiness; academic performance; adolescence 


\section{INTRODUCCIÓN}

El uso de celulares, computadoras, tabletas y otros dispositivos tecnológicos forman parte de la vida cotidiana de menores y adolescentes que han crecido en la era de las Tecnologías de la Información y la Comunicación (TIC). En efecto, este es el contexto tecnológico en el que se desenvuelven los adolescentes en Colombia, además de que se está proyectando según cálculos del Gobierno de Iván Duque, llegar al 70\% de la población conectada. Con todo esto el país se prepara para dar un paso más importante: La quinta generación de estándares de comunicaciones móviles, que ofrece una mayor velocidad y prestaciones avanzadas. Con esta red 5G, los usuarios pueden acceder a funciones móviles avanzadas como video llamadas, TV móvil y aplicaciones de banda ancha sin problemas de fiabilidad.

Ahora bien, por sí solas las TIC no suponen un riesgo, el uso problemático se plantea cuando su uso casi ininterrumpido impacta negativamente en el desarrollo normal de la vida cotidiana del adolescente; descuidando las actividades académicas, laborales y/o domésticas por estar más tiempo en el mundo 'virtual' que en el 'real' (Díaz-Vicario, Mercader y Gairín, 2019). De acuerdo con el boletín informativo realizado en Colombia por el Departamento Administrativo Nacional de Estadistica (DANE, 2019), según la última encuesta realizada sobre indicadores básicos de tenencia y uso de las TIC en hogares y personas en Colombia se obtuvo lo reflejado en la Tabla 1.

TABLA 1.

Total nacional de hogares en Colombia que disponen TIC.

\begin{tabular}{|c|c|c|c|c|}
\hline $\begin{array}{c}\text { Posee TV } \\
\text { convencional color, } \\
\text { LCD, plasma o LED }\end{array}$ & $\begin{array}{l}\text { Utiliza servicio de } \\
\text { televisión por cable, } \\
\text { satelital o IPTV }\end{array}$ & $\begin{array}{l}\text { Al menos una } \\
\text { persona tiene } \\
\text { teléfono celular }\end{array}$ & $\begin{array}{c}\text { Poseen } \\
\text { computadores }\end{array}$ & Conexión a internet \\
\hline El 90.7\%. & El $66.2 \%$. & $\begin{array}{l}\text { El } 80 \% \text { de los } \\
\text { cuales el } 78.1 \\
\text { inteligente. }\end{array}$ & $\begin{array}{l}\text { El } 28.8 \\
\text { portátil, el } 20.6 \\
\text { escritorio y el } \\
10.9 \text { tableta. }\end{array}$ & $\begin{array}{l}\text { El } 40.5 \% \text { fijo; } 29.2 \% \\
\text { móvil; } 83.1 \% \text { de } \\
\text { personas que usaron } \\
\text { internet, lo hacen } \\
\text { en su hogar; } 84.9 \% \\
\text { usan internet desde } \\
\text { telefonía celular; } \\
82.2 \% \text { usan internet } \\
\text { para redes sociales. }\end{array}$ \\
\hline
\end{tabular}

Fuente: DANE (2019).

En efecto, en el país el Ministerio de Tecnología de la información y la comunicación está encargado de promover y potenciar la realidad digital con la finalidad de reducir las brechas tecnológicas en diferentes sectores de la población, buscando la democratización, masificación, apropiación y uso de internet y las TIC (Orozco, Vásquez-Rizo y GabalánCoello, 2020), la finalidad es lograr un uso adecuado de las mismas sin afectar la calidad de vida de los usuarios. Sin embargo, aunque todo esto representa avances significativos para el desarrollo social, es importante señalar que, por sí solas, las Tecnologías de información y comunicación no suponen un riesgo, sino su uso desmedido, en el caso de los adolescentes, al ser este casi permanente, a impactando negativamente en el desarrollo normal de sus 
vidas; al descuidar sus responsabilidades, actividades académicas y/o domésticas por estar conectados virtualmente (Díaz-Vicario et al., 2019).

Es importante resaltar que al conocer los riesgos que implican la dependencia de la tecnología en la cotidianidad de los adolescentes, es menester la búsqueda de estrategias de prevención, donde los principales actores sean los padres de familia, docentes y administrativos, los cuales deben comprometerse en una cultura de buenas prácticas controladas, que además de nutrirles con información, establezcan estrategias educativas que involucren toda la comunidad educativa en el manejo de las TIC y en el caso de los padres su interacción más en estos contextos, para informarse de los peligro en los que pueda estar su hijo.

Por otro lado, en cuanto a los riesgos y oportunidades tecnológicas que experimentan los niños y adolescentes en su vida cotidiana, según un estudio realizado por la universidad EAFIT, Colombia, con la empresa Tigo a 458 niños y adolescentes, con un $50 \%$ de cada sexo, entre 9 y 16 años, pertenecientes a 43 colegios públicos y privados en las ciudades de Bogotá, Medellín, Bucaramanga, Cali, Barranquilla, Pereira, Cartagena y Manizales, se evidenció que el 84\% de los niños y jóvenes entre 9 y 16 años ya tienen perfiles en redes sociales, aunque lo permitido es a partir de los 13 años, gastan aproximadamente tres horas y media diarias navegando en las redes y esta cifra aumenta hasta 5 horas en los adolescentes (Zuluaga, 2019).

En todo caso, el 75\% de los menores se conectan a los celulares inteligentes desde sus habitaciones, lo que limita las restricciones por parte del adulto responsable otros se conectan mediante tabletas y computadores de uso propio; el 69\% usa dispositivos en casa de amigos y el $85 \%$ asegura que se conectan en zonas comunes de la casa; el $64 \%$ tiene permitido el uso de alguna red social; y, el 20\% ha dejado sus necesidades básicas diarias de lado por estar navegando en internet (Zuluaga, 2019). Por ende y, teniendo en cuenta lo descrito anteriormente, es importante destacar que los mercados digitales portan una idea de consumo, sustentada por la demanda de tecnología altamente sofisticada e inteligente, en permanente cambio y actualización (Rivoir y Morales, 2019).

Dentro de este orden de ideas, hay un concepto denominado brecha digital, el cual hace referencia al siglo XXI, en este sentido los hijos son nativos digitales y sus padres migrantes en este mundo, generando una distancia en estos dos grupos, siendo unos los que acceden a las Tecnologías de la Información y Comunicación (TIC) sin dificultad y los otros no. Uno de los orígenes de esta brecha es la pérdida de control que se ha generado por el uso del Wifi, el cual permite conexión a internet desde cualquier lugar de las viviendas fuera de la supervisión paterna, impactado esta situación de manera excepcional en los adolescentes debido a la vulnerabilidad que afrontan por su etapa crítica del desarrollo de la corteza prefrontal (Pedrero-Pérez et al., 2019).

En cuanto al sueño, hay que destacar que, niños, adolescentes y adultos requieren unas horas específicas de sueño para su adecuada funcionalidad. En el caso de los adolescentes, se requiere entre 9 y 10 horas y, al presentarse un retraso se afecta el despertar; en efecto, un gran porcentaje de adolescentes entre las edades de quince y diecisiete años duermen un promedio de siete horas durante la noche - cifra por debajo de lo recomendado- debido al uso constante de la tecnología, evidenciando un desajuste entre su sistema circadiano y su entorno en cuanto al periodo de sueño nocturno normal (Melendro, García y Goig, 2016). 
El sueño es un proceso activo, que aun cuando el cuerpo está en inactividad, el cerebro funciona; por eso se define como una acción endógena que se produce desde el interior mismo del organismo y debido a esta característica, es fundamental identificar los tres mecanismos básicos que lo coordinan y gobiernan tanto al sueño como a la vigilia. Estos mecanismos son: el equilibrio del sistema nervioso autónomo, los impulsos homeostáticos y por último los ritmos circadianos. Estos 3 mecanismos acompañados por la edad y los factores individuales de cada ser, generan el equilibrio y la adaptación en cualquier tiempo y permanencia (Torres y Monteghirfo, 2011).

Esta reducción de horas de sueño, se presenta por un fenómeno conocido como Vamping en donde los jóvenes pasan conectados utilizando sus aparatos tecnológicos durante toda la noche y madrugada, acciones realizadas durante un largo tiempo, sin supervisión de un adulto (Pastrana, Salgado y González, 2019). Del mismo modo, el sueño es un factor que influye directamente en el aprendizaje ya que es el regulador de la conducta, la emoción y la atención; por ello, la calidad y duración de este no sólo está ligado al aprendizaje, a la consolidación de la memoria y al rendimiento académico, sino también a las alteraciones asociadas producidas por un sueño inadecuado que puede desencadenar somnolencia diurna, afectando así la adecuada evolución del adolescente en la escuela (Araya Pizarro y Espinoza, 2020).

Santillán, Segovia y Saigua (2020) plantean que el uso de ordenadores, teléfonos móviles o Tablet en los adolescentes genera retrasos a la hora de levantarse y de acostarse, tanto en la semana escolar como en el fin de semana y, a este escenario, se le identifica como una tendencia hacia la vespertinidad, es decir, a la tipología circadiana o cronotipo caracterizado de manera individual por la capacidad de estar más activo y alerta en un periodo determinado del día; este desajuste es el responsable de la falta de sueño durante la semana escolar, la cual se intenta recuperar los fines de semana durmiendo el mayor número posible de horas, creando así una diferencia significativa entre los horarios de sueño de los días de semana y los horarios de sueño de los fines de semana.

En consecuencia, según Ríos-Flórez, López-Gutiérrez y Escudero-Corrales (2019) y Lugo (2019), un mal dormir tiene consecuencias negativas en el cuerpo y en el cerebro, derivando cansancio, disminución de la atención, concentración, memoria y rendimiento cognitivo; fatiga, somnolencia y alteraciones del humor, producto de la secreción de hormonas estresores, resultado de la falta de sueño. Por tal motivo, el sostener una buena higiene del sueño contribuye a un adecuado desarrollo cognitivo y un mejor rendimiento académico.

Además, en estudios realizados por Suardiaz-Muro et-al. (2020) y Lira y Custodio (2018), señalan la mejor calidad del sueño en los estudiantes como factor determinante en promedios de notas más elevados a diferencias de los que tienen una mala calidad de sueño, con menos de nueve horas y forma irregular de acostarse presentando estos una afección en su rendimiento académico. De modo que, las evidencias aportadas por estos estudios, recalcan la necesidad de examinar las posibles consecuencias del uso de las TIC en la franja nocturna de los adolescentes, análisis abordado desde la visión de profesores, orientadores, expertos y padres de familia. 


\section{Metodología}

Se empleó una metodología cuantitativa, con un diseño transeccional, no experimental de corte correlacional. La población objeto de estudio es de 307 estudiantes en nivel básica secundaria pertenecientes a una institución educativa en Valledupar (departamento del Cesar, Colombia).

\section{Participantes}

Las unidades de análisis están constituidas por 201 estudiantes de secundaria, seleccionados como muestra probabilística de tipo transversal, de la cual 102 hombres (50.7\%) y 99 mujeres (49.3\%), en edades de 13 a 18 años. Los criterios de inclusión utilizados para la selección de la muestra fueron estudiantes del novenos, décimo y undécimo, de educación básica secundaria. Dentro de las consideraciones éticas se tuvo en cuenta la aplicación del consentimiento informado a los padres de familia y el aval del comité de Bioética de Investigación de la Universidad Metropolitana, amparado en la normatividad legal vigente y las normas científicas, técnicas y administrativas establecidas para la investigación en seres humanos (Resolución 8430, 1993).

Como criterio de exclusión se tuvo en cuenta, los estudiantes que sus padres no firmaron el consentimiento informado, aquellos que al aplicársele el Test de Inteligencia Breve de Reynolds (RIST), obtuvieron un resultado $=$ o menor a 79 puntos, siendo indicador de afectación al nivel de inteligencia, y todos aquellos estudiantes no presentes el día de la aplicación de las pruebas. Una vez establecido el muestreo por inclusión y exclusión la población excluida es del 35\% quedando la muestra conformada por $(n=201)$ estudiantes, siendo el $65 \%$ de la población.

Dentro de las técnicas e instrumentos utilizados se encuentra: el Test de Inteligencia Breve de Reynolds (RIST) y, la prueba de Screening que permite obtener una estimación general del nivel de inteligencia de personas con edades comprendidas entre los 3 y los 94 años de edad y se utiliza en grandes grupos para evaluar el estado psicológico o cognitivo de sus participantes. Asimismo, está compuesto de dos subtest: adivinanzas (subtest verbal) y categorías (subtest no verbal), relacionado con la evaluación de la inteligencia fluida. Los baremos de la prueba están basados en una muestra de más de 2000 casos españoles (Santamaría y Fernández, 2009). La recolección de datos, en cuanto a la variable rendimiento académico, se obtuvo directamente de las notas académicas de los participantes que proporcionó la Institución Educativa.

De igual manera, se hace uso de la Escala de Somnolencia de Epworth para Niños y Adolescentes (ESS-CHAD), desarrollada en 2015 por el Dr. Murray W. Johns, ya que presento un análisis psicométrico establecido como una medida de somnolencia durante el día para adolescentes de 12 a 18 años de edad (Murray, 2015). Dicha escala revisa la predisposición de la persona de quedarse dormido en ocho distintas situaciones de la vida diaria; la puntuación global tiene un rango de 0 a 24, donde las puntuaciones mayores a diez son consideradas como positivas para padecer Somnolencia Diurna (SD) y, cuanto mayor sea la puntuación obtenida, mayor será la gravedad de la somnolencia. Hay un 
alto nivel de coherencia interna entre los ocho elementos del instrumento, medido por el a de Cronbach, que va desde 0.73 hasta 0.88 . Se ha demostrado ser válida, confiable y unidimensional.

Igualmente, para efectos de conocer el tiempo y uso de dispositivos tecnológicos en las franjas nocturnas utilizados por la muestra, más ciertos comportamientos y hábitos requeridos por la investigación que denoten la conducta de nuestra población de estudio, se diseñó un cuestionario con preguntas argumentadas por la revisión teórica de esta indagación y que son el soporte bibliográfico y argumentativo como respaldo para plantear la pregunta de investigación.

El cuestionario consta de veintiún preguntas repartidas así: seis relacionadas con el uso, tiempo e identificación de los dispositivos a los que tiene acceso la población de estudio y el contexto donde lo usan, tanto en la noche, como en los días de la semana y los fines de semana; ocho relacionadas con hábitos de conducta en las noches con el uso de estos dispositivos; tres relacionadas con las actividades que realizan habitualmente en las noches a parte del uso de dispositivos tecnológicos; y cuatro relacionadas con su actitud y efectividad en el desarrollo de sus actividades. Y, como criterio de validación del presente cuestionario, se solicitó a expertos evaluar el instrumento para su validación y aplicación a la muestra, con el objetivo de calcular la confiabilidad del cuestionario. También se le aplicó una prueba piloto a 300 sujetos que no forman parte de la muestra pero que poseen iguales características y, una vez tabulados los resultados en un cuadro de doble entrada, donde se registraron sujetos versus ítems, se recurrió al uso del coeficiente Alfa de Cronbach, obteniendo un índice de confiabilidad de 0.87 .

$$
A=21 / 20 \quad\left(\begin{array}{r}
1-\sum 27.29 \\
157.40
\end{array}\right)
$$

Y, según los criterios establecidos por Ruiz (2017), se traduce en un instrumento altamente confiable, con consistencia en las respuestas de los sujetos, lo cual se evidencia en la Tabla 2.

TABLA 2.

Escala para la interpretación de la confiabilidad.

\begin{tabular}{ll}
\hline \multicolumn{1}{c}{ Rango } & \multicolumn{1}{c}{ Interpretación } \\
\hline 0.81 a 1.00 & Muy Alta \\
0.61 a 0.80 & Alta \\
0.41 a 0.60 & Moderada \\
0.21 a 0.40 & Baja \\
0.01 a 0.20 & Muy Baja \\
\hline
\end{tabular}

Fuente: Elaboración propia (2020). 


\section{Procedimiento}

Para la selección de la muestra se realizó una entrevista a los directivos de la institución seleccionada en donde se esbozaron los objetivos del proyecto $\mathrm{y}$, mediante el conocimiento que ellos tenían de su población escolar, se determinó cuáles cursos se ajustaban a las variables de estudio. Luego, en una reunión de entrega de informes académicos con padres de familia, se presentó la investigación y el consentimiento informado, que al firmarlos permitían que los estudiantes participaran en el proyecto, resaltando su participación de manera voluntaria.

Se agendaron las fechas para la aplicación de los diferentes instrumentos. En primer lugar, se aplicó el RIST para descartar afectaciones a nivel de inteligencia en la población de estudio, quedando esta prueba como criterio de exclusión. Después, de manera grupal por cursos, se entregaron impresas la escala de Epworth y el cuestionario de uso de dispositivos tecnológicos a la muestra, con presencia de la psicóloga e investigadora a fin de resolver las dudas y asegurar la objetividad en el manejo de la información.

\section{RESUltados}

El análisis de resultados se realizó con el programa estadístico Stargraphic y SPSS. (Statistical Package for the Social Sciences), versión 18, con una $n=201$ estudiantes de educación básica secundaria. Una vez procesada la información, se presentaron y analizaron los resultados derivados de las bases de datos y se argumentó, de manera descriptiva, con el propósito de explicar el comportamiento de cada una de las variables, de acuerdo a los objetivos planteados.

En la Tabla 3 se evidencian los rangos de somnolencia de acuerdo a la escala, en donde de un $100 \%$, un $68.2 \%$ de los estudiantes presento un nivel de Somnolencia y solo un $31.8 \%$ presento baja probabilidad de somnolencia.

TABLA 3.

Somnolencia diurna de los estudiantes.

\begin{tabular}{lllll}
\hline & Frecuencia & \% válido & \% válido & \% acumulado \\
\hline $\begin{array}{l}\text { Baja probabilidad } \\
\text { de Somnolencia. }\end{array}$ & 64 & 31.8 & 31.8 & 31.8 \\
$\begin{array}{l}\text { Nivel medio de } \\
\begin{array}{l}\text { Somnolencia } \\
\text { diurna. }\end{array}\end{array}$ & 50 & 24.9 & 24.9 & 56.7 \\
$\begin{array}{l}\text { Nivel alto de } \\
\text { Somnolencia. }\end{array}$ & 82 & 40.8 & 40.8 & 97.5 \\
$\begin{array}{l}\text { Nivel superior de } \\
\text { Somnolencia. }\end{array}$ & 5 & 2.5 & 2.5 & \\
Total & 201 & 100.0 & 100.0 & \\
\hline
\end{tabular}

Fuente: Elaboración propia. 


\section{Uso de dispositivos tecnológicos}

El 100\% de hogares de la población de estudio reporta el uso de dispositivos tecnológicos y conexión a internet, además del uso de dispositivos electrónicos en las habitaciones de los adolescentes, lo cual genera su uso entre 1 y 6 horas promedios en las noches después de las 7 p.m., generando interrupciones del sueño y todos aquellos aspectos neurofisiológicos que se alteran con la exposición a estos dispositivos. Lo mencionado anteriormente, se evidencia en la Tabla 4 y Tabla 5, donde el 89\% de la población, requiere de ayuda para levantarse los días de clase.

TABLA 4.

Uso de dispositivos tecnológicos en las noches.

\begin{tabular}{|c|c|c|c|c|c|c|}
\hline $\begin{array}{l}\text { Preguntas de } \\
\text { la } 1 \text { a la } 5\end{array}$ & $\begin{array}{l}\text { Tiene } \\
\text { en su } \\
\text { hogar }\end{array}$ & $\begin{array}{l}\text { Lo usa } \\
\text { en su } \\
\text { cuarto }\end{array}$ & $\begin{array}{c}\text { Nivel } \\
\text { de uso. } \\
\text { Avanzado }\end{array}$ & $\begin{array}{c}\text { Nivel } \\
\text { de uso } \\
\text { experto }\end{array}$ & $\begin{array}{c}\text { Horas de uso en la } \\
\text { noche. 1-3 horas, } \\
\text { después de las } 7.00 \text { p.m }\end{array}$ & $\begin{array}{c}\text { Horas de uso en la } \\
\text { noche. 3-6 horas, } \\
\text { después de las } 7.00 \text { p.m }\end{array}$ \\
\hline Televisión & $100 \%$ & $64.2 \%$ & $34.8 \%$ & $34.8 \%$ & $31.8 \%$ & $12.4 \%$ \\
\hline Celulares & $98 \%$ & $91 \%$ & $50.2 \%$ & $29.4 \%$ & $43.8 \%$ & $24.9 \%$ \\
\hline $\begin{array}{l}\text { Acceso a } \\
\text { Internet }\end{array}$ & $95.5 \%$ & $77.6 \%$ & $44.8 \%$ & $25.9 \%$ & $41.8 \%$ & $29.4 \%$ \\
\hline Computador & $94.5 \%$ & $45.8 \%$ & $40.3 \%$ & $20.4 \%$ & $27.4 \%$ & $11.4 \%$ \\
\hline Videoconsolas & $41.8 \%$ & $21.9 \%$ & $15.9 \%$ & $16.4 \%$ & No reporta & No reporta \\
\hline
\end{tabular}

Fuente: Elaboración propia.

TABLA 5.

Horas de uso de dispositivos tecnológicos en días de clase y no clase.

\begin{tabular}{|c|c|c|c|c|c|c|}
\hline & $\begin{array}{c}\text { Hora de } \\
\text { acostarse } \\
\text { entre días de } \\
\text { clase }\end{array}$ & $\begin{array}{c}\text { Hora de } \\
\text { acostarse } \\
\text { días no de } \\
\text { clase }\end{array}$ & $\begin{array}{l}\text { Hora de } \\
\text { levantarse } \\
\text { día de } \\
\text { clase }\end{array}$ & $\begin{array}{c}\text { Hora de } \\
\text { levantarse } \\
\text { días de no } \\
\text { clase }\end{array}$ & $\begin{array}{l}\text { Se despierta } \\
\text { solo o lo } \\
\text { llaman días } \\
\text { de clase }\end{array}$ & $\begin{array}{l}\text { Se despierta } \\
\text { solo o lo } \\
\text { llaman días } \\
\text { de no clase }\end{array}$ \\
\hline Antes de 10.p.m. & $40.8 \%$ & $8 \%$ & & & & \\
\hline Entre 10 y 12 p.m. & $50.7 \%$ & $39.8 \%$ & & & & \\
\hline 12 p.m. a 2.00 A.m. & $8.0 \%$ & $41.3 \%$ & & & & \\
\hline Después de 2 a.m. & $0.5 \%$ & $10.9 \%$ & & & & \\
\hline Entre 4 y 5 a.m. & & & $65.7 \%$ & & & \\
\hline Entre 5 y 6 a.m. & & & $34.3 \%$ & $10.4 \%$ & & \\
\hline Entre 7 y 9 a.m. & & & & $47.3 \%$ & & \\
\hline Entre 9 y 12 m. & & & & $36.3 \%$ & & \\
\hline Después de 12 m. & & & & $6.0 \%$ & & \\
\hline Algún familiar & & & & & $54.7 \%$ & $7.0 \%$ \\
\hline Despertador & & & & & $34.3 \%$ & $2.5 \%$ \\
\hline Solo & & & & & $10.9 \%$ & $90.5 \%$ \\
\hline
\end{tabular}

Fuente: Elaboración propia. 
TABLA 6.

Vigilancia por los padres de los hábitos en horas nocturnas.

\begin{tabular}{lllll}
\hline & \multicolumn{1}{c}{ Frecuencia } & \multicolumn{1}{c}{$\%$} & \multicolumn{1}{c}{$\%$ válido } & \multicolumn{1}{c}{$\%$ acumulado } \\
\hline Nunca & 23 & 11.4 & 11.4 & 11.4 \\
A veces & 137 & 68.2 & 68.2 & 79.6 \\
Siempre & 41 & 20.4 & 20.4 & \\
Total & 201 & 100.0 & 100.0 & 100.0 \\
\hline
\end{tabular}

Fuente: Elaboración propia.

En la Tabla 5 y Tabla 6 se evidencia un 20.4\% de los padres supervisan las conductas de los adolescentes en las noches y que, en días de clases, las actividades que más realizan en las noches es ver televisión con un 43.3\%, seguido de utilizar el celular con un 31.3\%. Son muchos los padres que se muestran temerosos por el uso de las TIC, sin embargo, estos temores tienen origen por la pérdida de control sobre los contenidos digitales observados por sus hijos y las personas con quienes se relacionan mediante estas tecnologías. Considerando que el poco control se acentúa además con la disponibilidad del uso del sistema Wifi permanente. Al mismo tiempo, existe un doble discurso sobre la temática. Unos padres, insisten en su uso, porque consideran su importancia para sus hijos y otros protestan por la intensidad que le dan a su uso (Sánchez et al., 2015).

\section{Correlación de las variables}

A partir del análisis de la Figura 1 se evidencia como a mayor puntaje en la escala de somnolencia, el desempeño académico de la población es básico y/a menor puntaje de la escala, su desempeño es alto.

MEDIA +/- DESVIACION ESTANDAR

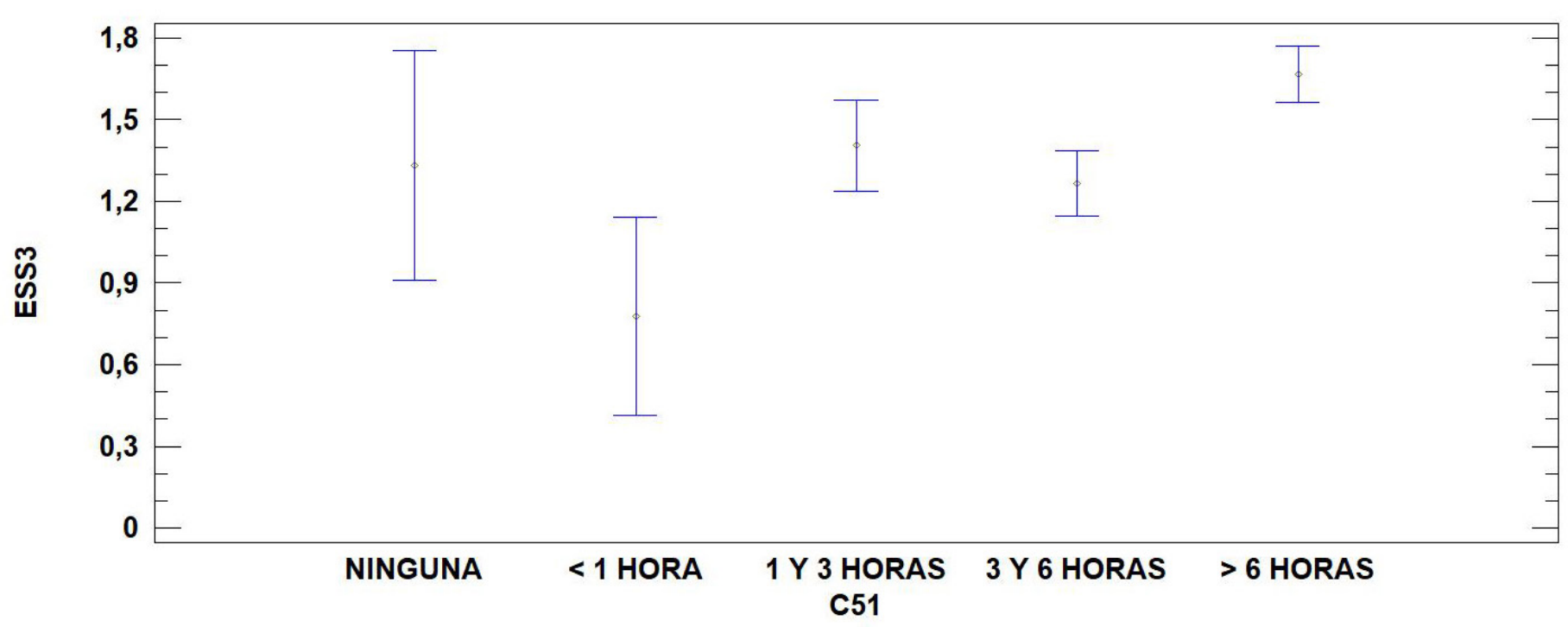

Figura 1. Somnolencia diurna con Rendimiento académico.

Fuente: Elaboración propia. 
En la Figura 1 se muestra que, entre más horas de dedicación al celular antes de dormir mayor es el grado de somnolencia cuando se esté sentado en el aula de clases en las mañanas y, aquellos que respondieron "NINGUNA", en cuanto al uso del celular y se dedican a ver televisión, navegar en internet o utilizar el computador, arrojan valores moderadamente altos de somnolencia cuando se esté sentado en el aula durante la mañana.

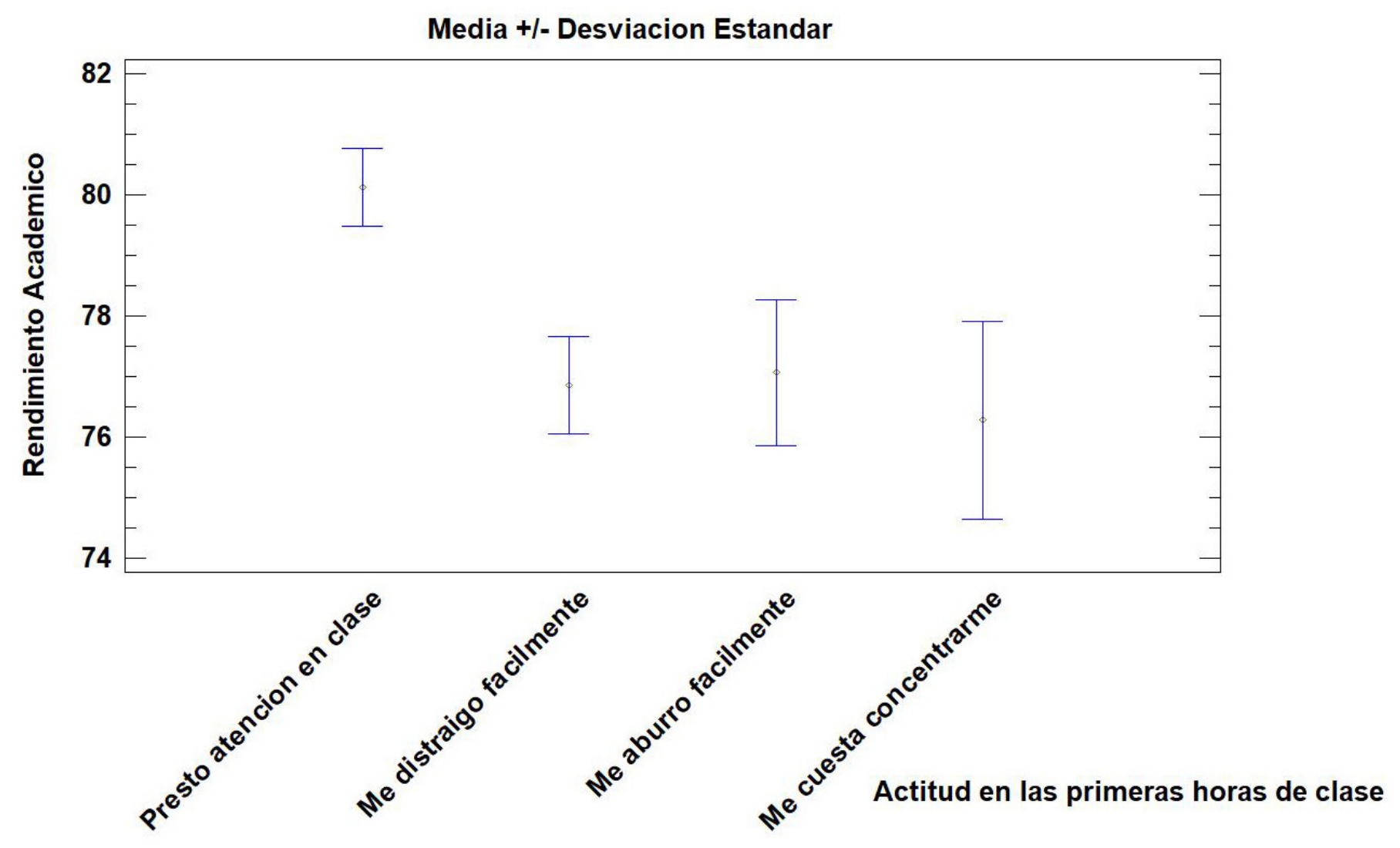

Figura 2. Actitud en las primeras horas de clase, con el rendimiento académico.

Fuente: Elaboración propia.

Asimismo, los estudiantes que más atención prestan en clase tienen un rendimiento académico alto por encima de los estudiantes que manifestaron en el cuestionario actitud de aburrimiento, distracción y dificultad de concentración en sus primeras horas de clase, evidenciando en la Figura 2 que el nivel de rendimiento académico, de estos últimos, está por debajo de los que si prestan atención. 
Media +/- Desviacion Estandar

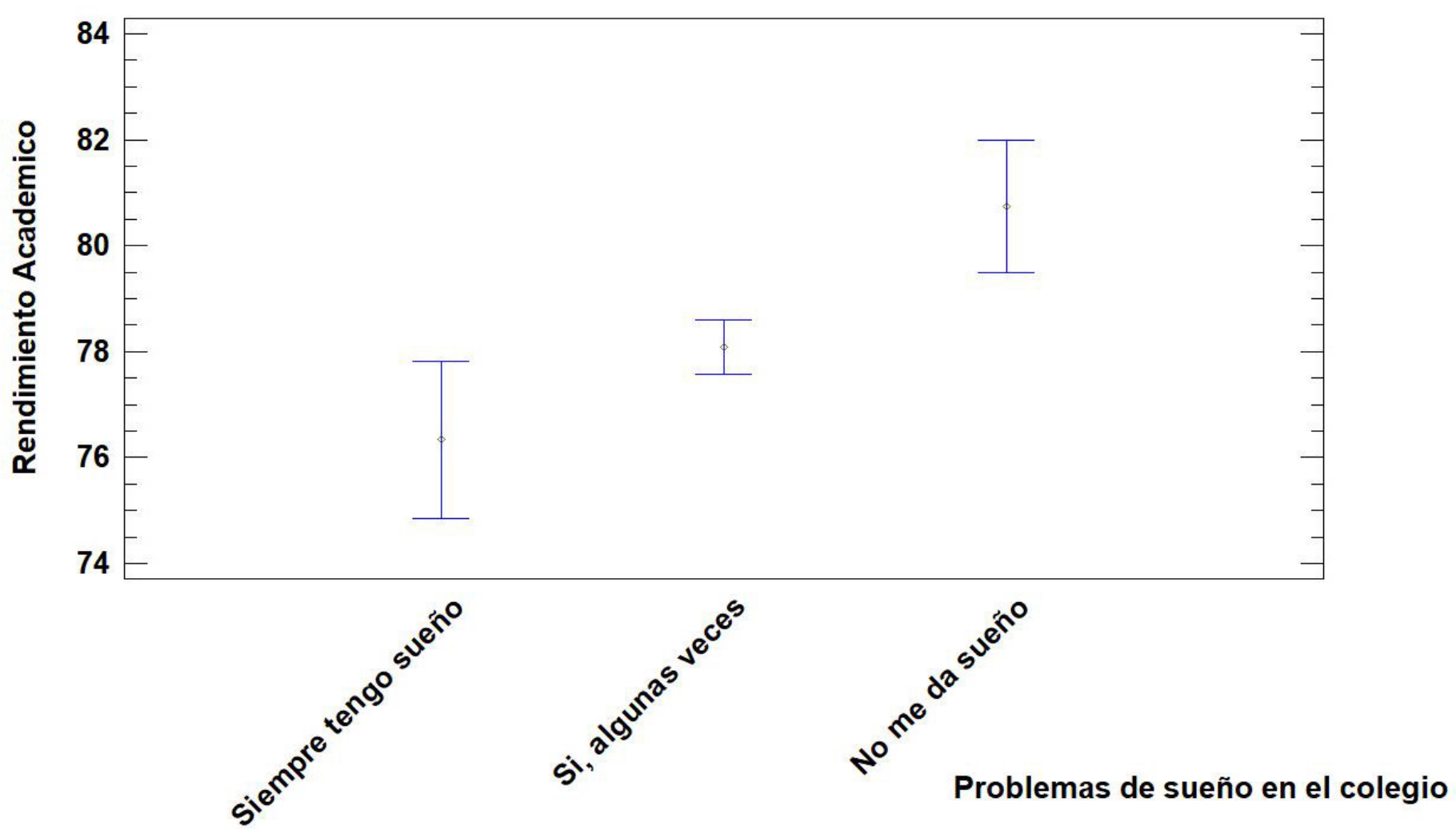

Figura 3. Rendimiento académico y problemas de sueño en el colegio.

Fuente: Elaboración propia (2020).

En la Figura 3 se evidencia los estudiantes declarar siempre tener sueño, su rendimiento académico está por debajo de los aquellos que señalaron algunas veces y estos con respecto a los que respondieron no me da sueño, su rendimiento está muy alto. Es decir, el rendimiento académico del estudiante es mejor, en la medida que no presentan problemas de sueño en el colegio.

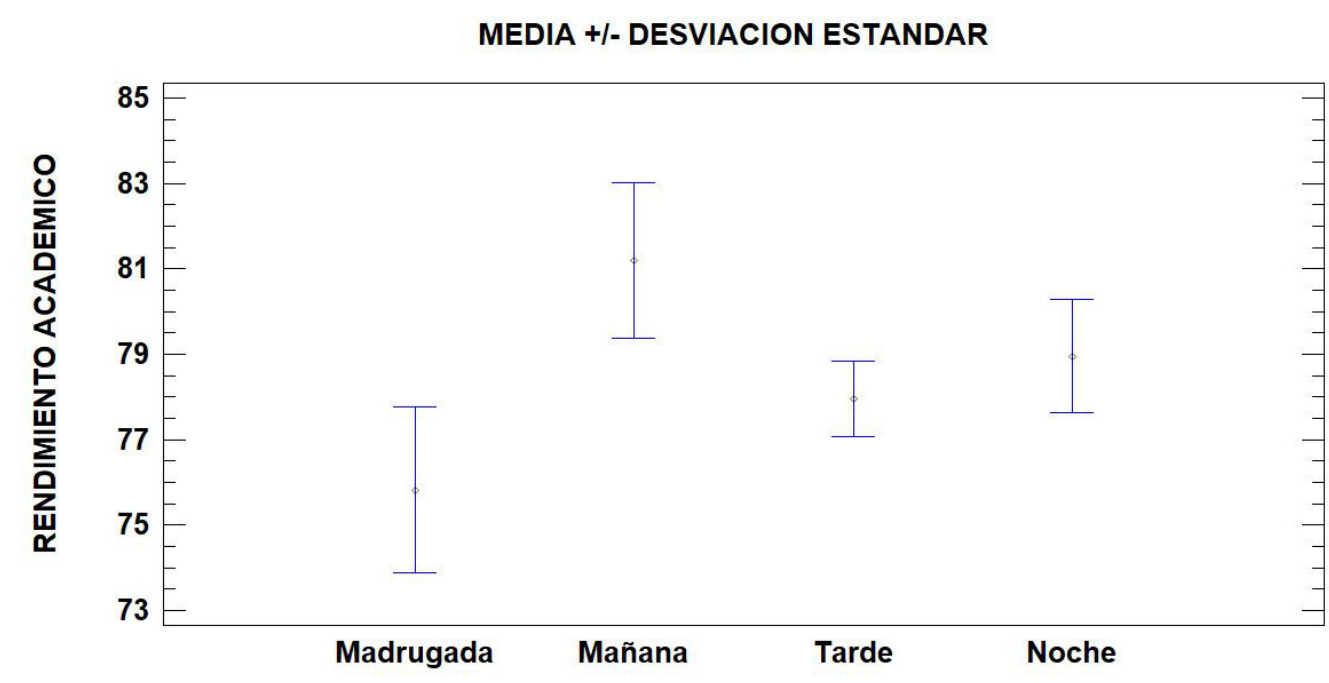

EN QUE MOMENTO DEL DIA TRABAJAS CON MAYOR EFECTIVIDAD

Figura 4. Momento del día que trabajo con mayor efectividad.

Fuente: Elaboración propia. 
De acuerdo con la Figura 4 los estudiantes que respondieron que el momento del día donde trabajan con mayor efectividad es la madrugada, se relacionan con los que presentan bajo rendimiento académico, al igual que los de la tarde y noche, diferente a los que manifiestan que sus horas del día con mayor efectividad es la mañana, estos tienen mayor desempeño académico, muestra de que su cronotipo es matutino a diferencia de los otros que son vespertino.

\section{DisCUSIÓN}

A partir de los hallazgos y, de acuerdo como lo plantean los estudios de Zuluaga (2019) y Ríos et al. (2019), se acepta el planteamiento presentado, estableciendo así que, si existe una relación entre el tiempo de uso de dispositivos tecnológicos en franja nocturna, con la somnolencia diurna y su afectación en el rendimiento académico de los adolescentes de un colegio de la ciudad de Valledupar (departamento del Cesar, Colombia).

En cuanto a las interrogantes tratadas se obtuvieron los siguientes resultados: en relación con el trabajo de los padres. El 74.9\% de las madres de la población de estudio, labora, de forma independiente o empleada, al igual que el 94.3\% de los padres. Es decir, que solo el $25.1 \%$ están bajo el cuidado y acompañamiento de sus madres.

Los adolescentes pasan más de 20 horas a la semana frente a una consola de videojuegos y utilizan estos dispositivos con destreza y sin esfuerzo, en su vida privada; esto se apoya en lo expuesto por Sánchez et al. (2015) al describir que estos dispositivos fortalecen la integración entre los adolescentes, los cuales pueden mantenerse conectados a cualquier hora del día o de la noche. Situación que se acentúa con la disponibilidad del uso del sistema Wifi, que les permite conectarse desde su habitación o en cualquier otro lugar fuera de la supervisión paterna (Castro-Sánchez, Zurita-Ortega, Martínez-Martínez, ChacónCuberos y Espejo-Gárces, 2016).

En relación con lo referido por estos estos autores el 100\% de hogares del estudio, cuentan con dispositivos electrónicos y acceso de ellos desde sus habitaciones, con conexión a internet y uso entre 1 y 6 horas promedios diarias. En cuanto a la edad de las personas que más utilizan dispositivos, según la Encuesta nacional de Tenencia de bienes TIC, realizada por el DANE en el 2017, arroja esta misma edad entre los 12 a los 18 años; con respecto a la falta de supervisión de los padres, nuestra investigación, indago en este tema y los resultados fueron que el $68.2 \%$ de estos padres a veces cuidan los hábitos de sueño de sus hijos, un $11.4 \%$ nunca lo hace y solo el $20.4 \%$ siempre vigila estos hábitos.

Lo referido a evidencias científicas que señalan los beneficios recibidos durante el sueño, el estudio no arroja estas evidencias, como resultado de un estudio experimental; en cambio Aguilar et al. (2017) cuentan con evidencias científicas donde señalan que durante el sueño REM, se favorece la memoria procedimental (Repetición de un acto motor) y en el NREM se beneficia la memoria declarativa (recordar experiencias pasadas) es decir en el artículo no se ofrece esta información. Lo mismo ocurre con Laberge et al. (2001), quienes indagaron acerca de las alteraciones que se dan a nivel de atención, concentración, memoria, razonamiento, habilidades psicomotoras cuando hay cambio en el horario de sueño al menos en dos horas o insuficiencia en este, encontrando que estas acciones promueven el bajo rendimiento escolar, así como el incremento de la irritabilidad, ansiedad y depresión. 
En relación con las causas de la mala calidad del sueño, Dewald, Meijer, Oort, Kerkhof y Bögels (2010) plantean que en ocasiones esto se debe al tiempo expuesto con la luz artificial (luz que irradia un monitor de computadora, o un móvil) y que tiende a disminuir la cantidad de melatonina segregada, impidiendo al adolescente darse cuenta de que necesita dormir a lo que Shochat, Flint-Bretler y Tzischinsky (2010) señalan que los niños y adolescentes que tiene televisor u ordenador en su habitación muestran una mayor latencia de sueño y tienden a dormir menos horas y a retrasar la hora de acostarse.

Lo que describen estos autores en sus escritos son argumentos que concuerdan con los resultados de esta investigación, en los hallazgos se encontró que el 100\% de hogares cuentan con diversos dispositivos electrónicos y conexión a internet, con múltiples dispositivos en las habitaciones y uso entre 1 y 6 horas promedios diaria después de las 7 de la noche, lo que puede en determinado momento generar interrupción del sueño y con esto todos aquellos aspectos neurofisiológicos que se alteran con la exposición a estos dispositivos.

Los mayores porcentajes de acostarse de nuestra población de estudio, están entre las 10 p.m. y 12 de la noche los días de la semana, y el porcentaje más alto de horas para levantarse esta entre las 4 a.m. a 5 a.m., seguido con el hecho de que el mayor porcentaje de la población requiere que los llamen para levantarse, para poder cumplir con su responsabilidad de ir al colegio. Caso contrario sucede los fines de semana, donde los porcentajes altos de acostarse, están entre las 12 a.m. a 2.00 a.m. y hora de levantarse el promedio más alto esta entre las 9 a.m. a 12 del mediodía, con un porcentaje alto que se levanta solo, sin la ayuda de nadie o nada.

En cuanto a la relación del sueño y la actitud en las primeras horas de clase, el uso de los dispositivos electrónicos de manera nocturna afecta el rendimiento académico. En esta investigación, la pregunta sobre la actitud en las primeras horas de clase con el rendimiento académico, se evidencia que a mayor atención del estudiante en clase, mayor será su rendimiento académico, entre más se distrae, aburre o no se concentra, más bajo será su rendimiento académico, resultados acordes con los expresado por Masalana, Sequeida y Ortiz (2013) donde describe que el 60\% de adolescentes, se sienten cansados $\mathrm{y}$ fatigados al no promover los descanso requerido para su organismo, en especial para el sistema nervioso que se restablece o repara durante el sueño; lo que repercute en su calidad de vida, deserción escolar, ausentismo, bajo nivel de rendimiento intelectual, alteración en la memoria, labilidad emocional (cambiar abruptamente el estado emocional), agresividad, irritabilidad, baja tolerancia a la frustración, alteraciones que se ven reflejadas por un desarrollo anormal del sueño, afectando funciones orgánicas y psicológicas.

En cuanto al desempeño académico, el Ministerio de Educación Nacional en Colombia (MEN) estipula unas áreas obligatorias y fundamentales para el logro de los objetivos.

Entonces según los resultados obtenidos por las bases de datos de nuestra institución, contexto de la investigación, el programa académico de los tres cursos a los que hace parte la población de estudio, está conformado por 17 asignaturas, de las cuales 16 pertenecen a estas áreas bases y solo 3 de ellas se encuentran en el nivel de desempeño alto, a saber, artes, ética y educación física, las otras 13 se encuentran en desempeño 
básico, según la escala de valoración suministrada por este ente nacional. Si comparamos estos resultados con la prueba PISA, que realiza la OCDE, en donde sus áreas de evaluación son ciencias, lengua castellana y Matemáticas, se interpreta entonces que ninguna de estas tres áreas se encuentra en el nivel alto y superior. Resultados que evidencian una razón por la cual no se alcanza el promedio mínimo que requiere el nivel básico de PISA.

En razón de lo anterior el presente estudio muestra la innovación de la temática y como se requiere de investigaciones que apunten a identificar factores cruciales que podrían estar afectando el rendimiento académico: países de Europa, están apuntando a estas investigaciones, como es el caso de España, que según la encuesta llevada a cabo por la Unidad Valenciana, develan que el adolescente debe dormir un promedio de 9 horas, para satisfacer las necesidades del organismo, pero la mitad de ellos duerme el 20\% menos; Este déficit puede perjudicar su rendimiento académico y su desarrollo, además de originar problemas de hiperactividad e impulsividad, cambios en el estado de ánimo y mayor riesgo de accidentes de tráfico; además de esto, los datos estadísticos demuestran que en el sueño, "menos del 50\% de los niños de entre 6 y 12 años de edad se duermen de manera autónoma, un $27 \%$ tienen problemas a la hora de irse a la cama, un $11.3 \%$ tardan demasiado tiempo en dormirse (más media hora), un 6.5\% presenta despertares nocturnos, un $17 \%$ se despierta con dificultad por la mañana y otro $17 \%$ sufre fatiga relacionada con alteraciones del sueño.

Explicaciones que se relacionan con los resultados de la escala de somnolencia de nuestra población de estudio y que, según la tabla de puntuación, ellos están entre 8 y 9 puntos, es decir, presentan una cantidad media de sueño durante el día. Que inicia desde 8.75 puntos en noveno, va subiendo a 9.29 puntos en $10^{\circ}$, y sigue en ascenso en $11^{\circ}$ con 9.46 puntos, indicador de un alto nivel de somnolencia. De aquí la importancia de tomar control, como lo argumenta Gutiérrez (2012) cuando narra que el comportamiento cotidiano del adolescente, es aislarse en sus habitaciones y en vez de dormir, navegan por internet, ven televisión, hablan por teléfono, usan videojuegos, generando una relación directa entre el sueño corto y el uso de aparatos tecnológicos inmediatamente antes de acostarse. Situaciones que no controlan sus padres y los lleva a la tendencia del cronotipo vespertino y retraso de fase, que los condiciona a la aparición de la Somnolencia Diurna Excesiva (SDE).

Y cuando se habla de cronotipo vespertino, con respecto a la pregunta, momento del día en el que trabajas con mayor efectividad, el 53,2 \% respondió que, en la tarde, seguido de un $23.4 \%$ en la noche, con un $10.9 \%$ en la madrugada, es decir que solo un $12.4 \%$ de esta población tiene mayor efectividad en la mañana, a lo que apunta el cronotipo matutino. Con todo esto se debe agregar que son pocos los estudios que han referenciado la relación de la somnolencia diurna y su afectación en el rendimiento académico, por uso de dispositivos tecnológicos en franja nocturna, en población de estudiantes de educación básica obligatoria. De aquí la importancia de nuevos estudios de intervención dirigidos a disminuir los comportamientos de los adolescentes en relación al manejo de los dispositivos electrónicos de manera nocturna con la finalidad de mejorar el rendimiento académico en el país. 


\section{CONCLUSiOnes}

El sueño es una de las necesidades del ser humano que tiene una relación con la calidad de vida de las personas y tiene una influencia significativa en el rendimiento cognitivo. Por ende, es menester cuidar de su buena calidad para mejorar el rendimiento académico y desarrollar campañas de difusión y concientización para lograr que todo individuo en general se ocupe por tener una buena calidad de sueño, sobre todo conociendo desde esta investigación los factores que incrementan el compromiso cognitivo de los adolescentes en este caso el mal uso de dispositivos tecnológicos en franja nocturna.

Ahora bien, no se debe olvidar que el acompañamiento a esta tipo de conducta, frente a sus actividades nocturnas, no es solo por capricho y control por la edad por parte de los padres $u$ otros sino que se encuentran en una etapa en la que todavía no se ha terminado de desarrollar en su totalidad la corteza prefrontal, responsable de habilidades como planificar, establecer prioridades y control.

Finalmente, con base a las evidencias dadas en este estudio se exhorta a los padres a mantener una actitud más vigilante y persuasiva con sus hijos adolescente en relación a sus horas de descanso.

\section{REFERENCIAS}

Aguilar, L., Caballero, S., Ormea, V., Salazar, G., Loayza, L. \& Muñoz, A. (2017). La importancia del sueño en el aprendizaje: visos desde la perspectiva de la neurociencia. Avances en Psicología, 25(2), 129-137. Recuperado de http://revistas.unife.edu.pe/index.php/avancesenpsicologia/article/view/349/80

Araya-Pizarro, S. C. y Espinoza, L. (2020). Aportes desde las neurociencias para la comprensión de los procesos de aprendizaje en los contextos educativos. Propósitos y Representaciones, 8(1), 1-10. https://dx.doi.org/10.20511/pyr2020.v8n1.312

Castro-Sánchez, M., Zurita-Ortega, F., Martínez-Martínez, A., Chacón-Cuberos, R. y Espejo-Gárces, T. (2016). Clima motivacional de los adolescentes y su relación con el género, la práctica de actividad física, la modalidad deportiva, la práctica deportiva federada y la actividad física familiar. $R I C Y D E, 12(45), 262-277$. http://dx.doi. org/10.5232/ricyde2016.04504

Dewald, J. F., Meijer, A. M., Oort, F. J., Kerkhof, G. A. \& Bögels, S. M. (2010). The influence of sleep quality, sleep duration and sleepiness on school performance in children and adolescents: a meta-analytic review. Sleep medicine reviews, 14(3), 179-189. https://doi.org/10.1016/j.smrv.2009.10.004

Díaz-Vicario, A., Mercader, C. y Gairín, J. (2019). Uso problemático de las TIC en adolescentes. Redie, 21, e071-e0711. https://doi.org/10.24320/redie.2019.21.e07.1882

Gutiérrez, D. (2012). Privación crónica de sueño en adolescentes universitarios y su influencia en el rendimiento académico. [Monografia]. Universidad Militar Nueva Granada, Bogotá, D.C., Colombia. Recuperado de http://repository.unimilitar.edu.co/bitstream/10654/9960/2/GutierrezDiazDianaPaol a2012.pdf 
Laberge, L., Petit, D., Simard, C., Vitaro, F., Tremblay, R. \& Montplaisir, J. (2001). Development of sleep patterns in early adolescence. Journal of Sleep Research, 10(1), 59-67. https://doi.org/10.1046/j.1365-2869.2001.00242.x

Lira, D. y Custodio, N. (2018). Los trastornos del sueño y su compleja relación con las funciones cognitivas. Revista de Neuro-Psiquiatría, 81(1), 20-28. https://doi.org/10.20453/ rnp.v81i1.3270

Lugo, A. (2019). El rendimiento académico y el sueño en la Educación Media Superior: el caso de un bachillerato en Ciudad Juárez. [Tesis de Posgrado]. Universidad Autónoma de Ciudad de Juárez, México. Recuperado de http://erecursos.uacj.mx/bitstream/handle/20.500.11961/5528/tesis\%20final-2019-alr-171403.pdf?sequence=1\&isallowed=y

Masalán, M., Sequeida, J. y Ortiz, M. (2013). Sueño en escolares y adolescentes, su importancia y promoción a través de programas educativos. Revista Chilena de Pediatría, 84(5), 554-564. http://dx.doi.org/10.4067/S0370-41062013000500012

Murray, J. (2015). Escala de Somnolencia de Epworth (ESS-CHAD). [Online]. Disponible en https:/www.merckmanuals.com/medical-calculators/EpworthSleepScale-es.htm

Melendro, M., García, F., \& Goig, R. (2016). El uso de las tic en el ocio y la formación de los jóvenes vulnerables. Revista Española de Pedagogía, 74(263), 71-89. Disponible en https://revistadepedagogia.org/lxxiv/no-263/el-uso-de-las-tic-en-el-ocio-y-la-formacionde-los-jovenes-vulnerables/101400009802/

Orozco-García, M., Vásquez-Rizo, F. y Gabalán-Coello, J. (2020). Incorporación, uso y apropiación social de las TIC para una educación de calidad. Una propuesta. Cultura Educación y Sociedad, 12(1), 47-62. https://doi.org/10.17981/cultedusoc.12.1.2021.04

Pastrana M. G., Salgado M. E. y González, E. S. (2019). Repercusiones cognitivo-conductuales de la práctica del vamping en adolescentes de décimo grado del Instituto Reino de Suecia de Estelí, período 2018-2019. [Tesis de Pregrado]. Universidad Nacional Autónoma de Nicaragua, Nicaragua. Disponible en https://repositorio.unan.edu. ni/11386/

Pedrero-Pérez E. J., Mora-Rodríguez, C., Rodríguez-Gómez, R., Benítez-Robredo, M. T., Franco A. O., González-Robledo, L. y Méndez-Gago, S. (2019). Síntomas prefrontales asociados al uso problemático de las Tecnologías de la Información y la Comunicación (TIC) en adolescentes. Revista internacional de psicología clínica y de la salud, 27(2), 257-273. Recuperado de https:/www.behavioralpsycho.com/wp-content/ uploads/2019/10/05.Pedrero-27-2oa.pdf

República de Colombia. DANE. (2019). Indicadores básicos de TIC en Hogares. [Boletín Técnico]. Disponible en https://www.dane.gov.co/index.php/estadisticas-por-tema/tecnologia-e-innovacion/tecnologias-de-la-informacion-y-las-comunicaciones-tic/indicadores-basicos-de-tic-en-hogares

República de Colombia. Ministerio de Salud. (4 de octubre de 1993). Por la cual se establecen las normas científicas, técnicas y administrativas para la investigación en salud. [Resolución 8430]. Recuperado de https://www.urosario.edu.co/Escuela-Medicina/Investigacion/Documentos-de-interes/Files/resolucion_008430_1993.pdf 
Ríos-Flórez, J. A., López-Gutiérrez, C. R. y Escudero-Corrales, C. (2019). Cronobiología del sueño y su influencia en la función cerebral. Cuadernos de Neuropsicología, 13(1), 12-33. Disponible en https://www.cnps.cl/index.php/cnps/article/view/351

Rivoir, A. L. y Morales, M. J. (2019). Tecnologías digitales: miradas críticas de la apropiación en América Latina. Buenos Aires: CLACSO. Recuperado de http://biblioteca. clacso.edu.ar/clacso/se/20191128031455/Tecnologias-digitales.pdf

Ruiz, C. (2017). Instrumentos de Investigación Educativa. Procedimientos para su Diseño y Validación. Barquisimeto. Editorial Brujas.

Santillán, E., Segovia, S. y Saigua, V. (2020). Relación de la duración del sueño y rendimiento académico en alumnos de la Unidad de Admisión y Nivelación. La Ciencia Al Servicio de la Salud, 11(1), 16-24. Disponible en http://revistas.espoch.edu.ec/index. $\mathrm{php/cssn/article/view/465}$

Sánchez, L., Crespo, G., Aguilar, R., Bueno, F.-J.., Benavent, R. y Valderrama, J. (2015). Los Adolescentes y las Tecnologías de la tecnología y la Comunicación (Tic). Valencia: Ayuntamiento de Valencia. Recuperado de: http://digital.csic.es/bitstream/10261/132633/1/ TICPadres.pdf

Santamaría P. y Fernández, I. (2009). Test de Inteligencia Breve de Reynolds RIST. Madrid: TEA Ediciones. Recuperado de http://www.web.teaediciones.com/Ejemplos/Extracto_manual_RIAS_RIST.pdf

Shochat, T., Flint-Bretler, O. \& Tzischinsky, O. (2010). Sleep patterns, electronic media exposure and daytime sleep-related behaviours among Israeli adolescents. Acta Pcediatrica, Referencias, 99, 1396-1400. https://doi.org/10.1111/j.1651-2227.2010.01821.x

Suardiaz-Muro, M., Morante-Ruiz, M., Ortega-Moreno, M., Ruiz, M. A., Martín-Plasencia, P. y Vela-Bueno, A. (2020). Sueño y rendimiento académico en estudiantes universitarios: revisión sistemática. Revista de Neurología, 43(53), 2-22. Recuperado de https:/www.svnps.org/wp-content/uploads/2020/09/rendimientoby020043.pdf

Torres, V. y Monteghirfo, R. (2011). Trastornos del sueño. Archivos de Medicina Interna, 33(Supl 1), S01-S46. Disponible en https://biblat.unam.mx/es/revista/archivos-de-medicina-interna/articulo/trastornos-del-sueno

Zuluaga, M. (2019). Un campus amigable con el planeta. Revista Universidad EAFIT, 54(173), 26-29. https://publicaciones.eafit.edu.co/index.php/revista-universidad-eafit/ article/view/5731

*Artículo de investigación científica derivado del proyecto de investigación titulado: "Relación entre el tiempo de uso de dispositivos tecnológicos en franja nocturna con la somnolencia diurna y su afectación en el rendimiento académico de adolescentes en Valledupar.

Francis Araque Barboza es Sociologa, Magister, Doctora en Ciencias Humanas. Docente a nivel de pregrado, postgrado y doctorado. Investigadora principal de proyectos de investigación activos. Coordinadora del área de investigación del programa de psicología de la Universidad Metropolitana. Investigadora Senior clasificada por Minciencias en Colombia. https://orcid.org/0000-0001-7420-520X 
Elisama Beltrán de La Rosa es Psicóloga egresada de la Universidad Metropolitana, realizando estudios pos graduales en universidades de Colombia, Europa y los Estados Unidos. Investigadora categorizada por Colciencias, investiga en el área clínica, con una línea base en VIH, Enfermedades crónicas y huérfanas. https://orcid.org/0000-0002-3119-2944

Olaiza Lobato Pérez es Psicóloga de la Universidad Nacional Abierta y a Distancia, Neurorehabilitadora Cognitiva e Investigadora. Maestrante en Ciencias de la Salud de la Universidad Metropolitana. https://orcid.org/0000-0001-7428-7268 